not entitled to assume this. Illuminating gas and other gases which give rise to carbon monoxide coma are not pure carbon monoxide, but contain many other carbon compounds, and there is some evidence that these are more toxic to nerve tissue than carbon monoxide.

In $1922 \mathrm{H}$. W. Haggard in the Amer. Jl. of Physiol., Vol. LX, published the account of a very interesting series of experiments bearing on this point.

He had shown that it was possible to take the neuroblasts from an early chick embryo and to grow them in suitable media, in hanging drop culture. He found that grown over air, 86 per cent. of the cultures lived; he then replaced the air by a mixture of gases in which carbon monoxide took the place of the atmospheric nitrogen. In this case 90 per cent. of the cultures developed, showing that carbon monoxide was not unfavourable to the developing neuroblasts.

When he admitted a very small amount of illuminating gas to the chamber (one part in a thousand) all the cultures died at once, showing that there was some very active poison in the new mixture; in so far as I am aware, there has been no further attempt to discover which of the various elements of illuminating gas are specially poisonous to nerve cells, but these experiments are sufficient to make it improbable that all the toxic effects are due to carbon monoxide.

I am indebted to Dr. David Kerr of St. James's Hospital for the notes of the medical condition of $\mathrm{E}$. $\mathrm{H}$. throughout his stay in the hospital.

\title{
A CLASSIFICATION OF CASES OF CONCOMITANT STRABISMUS BASED ON THE AETIOLOGICAL FACTOR
}

\author{
BY
}

\author{
M. A. PUGH
}

LONDON

IN the evolution of the treatment of squinting there have arisen from time to time, various theories as to its cause. Each new theory claimed to replace ideas previously held.

Thus, in 1827, Anthony White suggested that as a squint could be cured by a myotomy the original defect was entirely muscular in origin. In 1864 Donders pointed out the connection between hypermetropia and convergent squint, and between myopia and 
divergent squint, asserting that the deviation was due to a defect in the accommodation-convergence link. Javal in 1864 and Worth later in 1903, pointed out that some hypermetropes develop a divergent squint and some myopes a convergent squint and that a certain proportion of squinters are emmetropic. They held, therefore, that the accommodation-convergence link was not the primary factor, but that a defect of the fusion faculty was the only feature common to all squints. Still later in 1928 Inman suggested that psychological instability or psychological trauma was one of the essential causes.

It would seem that each theory is tenable provided that it is advanced for a particular group of squinters. The problem for the ophthalmologist is to make a more exact classification of cases of squint.

Worth's book on squint, 1902, laid the foundation for the presentday orthoptic training. He pointed out that by developing the fusion faculty in squinting eyes full binocular vision may be established, and that by using this binocular function the eyes may be trained to a straightness which is maintained under all conditions.

When cases of strabismus are examined for binocular vision for the first time different results are obtained. A case which has had a squint since birth will have very little binocular vision. A case in which the onset of the squint occurred at 5 years of age or later will often have a good fusion sense and even some stereoscopic vision at the angle of squint although the deviation may have been constant for five or more years. One girl, aged 18 years, had had a $20^{\circ}$ alternating squint since early childhood. She was operated upon without any previous training and the day the bandages were removed she was found to have good stereoscopic vision. Her eyes may have been occasionally straight when she was a child and so she may have developed stereoscopic vision but she gave no history of such relapses to straightness, even on direct questioning. We may find any grade of binocular vision in cases of squint of many years' duration. This suggests that in some cases the defect in fusion may be the result of the deviation and not always the cause of the squint. Generally cases of late onset have the more fully developed binocular vision.

There are exceptions to the theory that a refractive error is the primary factor, and there are exceptions to Worth's theory that a defect of fusion is the fundamental error; although these theories afford a satisfactory explanation of the origin of many squints.

There remains a group which may be emmetropic and possess Grade III binocular vision and yet a squint develops.

We find that the cases under training fall into four groups.

The first group includes the patients with a definite refractive error who are much improved with their glasses and are unstable 
without. They may have very poor or fairly good binocular vision but the main factor appears to be the ametropia.

The second group includes the cases of early onset in which the fusion faculty is very defective. These cases may have refractive errors, but they give the impression of having no idea of using both eyes together and their glasses do not make such a marked difference as in Group I cases.

The third group includes cases which may have a refractive error or may be emmetropic; they may have poor binocular vision or good stereoscopic vision, but they will give a history of having maintained straight eyes until some psychological disturbance gave rise to nervous instability which manifested itself as a squint.

The fourth group where some physical defect is present does not present difficulties in diagnosis, but sometimes insuperable difficulties in training.

An analysis of 500 cases gave the following results :-

\begin{tabular}{lllll} 
& & & \multicolumn{3}{c}{ Per cent. } \\
1. & Refraction error squints & $\ldots$ & $\ldots$ & 62 \\
2. & Fusion defect squints & $\ldots$ & $\ldots$ & 15 \\
3. & Psychological squints & $\ldots$ & $\ldots$ & 21 \\
4. & Physical defect squints & $\ldots$ & $\ldots$ & 2
\end{tabular}

\section{Refractive Error Squints}

These are the squints which fit in with Donders' theory. The hypermetropes converge and the myopes diverge.

The squint may be either unilateral or alternating. In treating a unilateral squint, the vision in either eye must be equalized by occlusion and the squint is often converted into the alternating type in young patients.

The difference stressed by some observers between true and acquired alternation does not seem to be marked in the reaction to training. A true alternating squint alternates spontaneously and it is often found that each eye is used to fix a particular portion of the field of vision so that as the gaze is directed from side to side first one eye and then the other takes up fixation, a quick alternation being produced. On the other hand an alternating squint developed in a previously unilateral squint as a result of occlusion uses the fixing eye over the whole field of vision for an appreciable period of time before taking up fixation with the other eye at will. Nevertheless, true alternating squints can develop firm fusion and stereoscopic vision. If a patient with a true alternating squint of a high degree is taught to fuse at the angle of deviation before operative measures put the eyes straight, the ultimate result tends to be much better than in an untrained case. The images are fused and the depth value of the objects around is appreciated. The 
eyes in this case will probably remain straight and controlled during all movements. Where no training has been undertaken before the operation the patient may, in fortunate cases, learn to fuse by his own efforts, but more often the images confuse him and he inclines to recover some degree of deviation of his visual axes in order to alternate again.

Although much can be done by training towards re-establishing a normal convergence-accommodation link the question of the spectacles remains an important one. In a convergent hypermetrope the full atropine correction is well tolerated as a rule and gives the most help. If such a case is put straight by operation these lenses may by reducing the accommodative effort, lead to a tendency to divergence. The best correcting lens is that which gives the best vision with fusion at zero in distant vision. Training does much to stabilize the convergence during accommodation, but in "refractive error" squints the correct spectacles are essential.

\section{Fusion Defect Squints}

This type corresponds to Worth's group of squints.

A patient with a squint and good vision in either eye should have diplopia, in a divergent squint the images should be crossed. A small percentage of cases have a constant true diplopia. A much larger percentage will give a history of diplopia at the onset of the condition if questioned carefully. Young children of two years of age may complain of seeing two mothers.

Most of the cases in the group do not have diplopia. They avoid this confusing symptom by one of two methods, suppression or false projection. If the suppression is confined to one eye, the eye becomes more or less amblyopic. If either eye is suppressed when the other eye is functioning an alternation of fixation occurs.

The larger proportion of cases develop a false projection-not a false macula, but a false localization of an object seen by either eye.

If a patient with a convergent squint of $20^{\circ}$ is placed in front of a Maddox scale and a rod is placed in front of the convergent eye, the patient may say that the line goes through the light or very near it and nothing will persuade him to change that statement.

If such a patient looks into an amblyoscope he will put the bird in the cage at zero or somewhere nearer to zero than his deviation justifies. Pictures may be fused in this false position and in cases of quick alternation some depth value may be obtained. The patient's desire for binocular vision is strong enough to allow him to fuse images even allowing for the deviation of the visual axes. The mechanism by which this result is obtained appears to be mostly cerebral, but there is a specialized hypersensitive zone lying 
between the macula and the false projection spot. In an alternating squint the spot on the retina of the diverging eye which corresponds in position to the macula of the fixing eye appears to develop a special localizing power in conjunction with the opposite macula. It has very little more visual acuity than the surrounding retina and very much less than the true macula. There is, nevertheless, a sensitized band from the false projection spot to the macula. If an image is brought in from the periphery to that area of retina no details can be made out until the macula is reached, but if the pictures are moved centrifugally the image remains clear longer than in a normal eye.

Thus we have three states, diplopia, suppression, and false projection. Diplopia is favourable and unless the squint is a large one the patient is easily trained to fuse the images on the orthoptic instruments and later does the same with surrounding objects, thereby straightening his eyes. If an operation is now performed no difficulty arises, the patient will fuse his images with very little training and will readily correct any residual deviation. The cases of suppression are more difficult, but if treated by occlusion before the eye is irrevocably amblyopic the patients usually can be trained to accept the diplopia and can be treated from that point as above. False projection gives rise to difficulties in straightening squinting eyes by any method. A patient with $20^{\circ}$ of false projection who has his eyes put straight by surgical measures will complain of diplopia as soon as the bandages are removed. This is usually a "false diplopia" as his images are falling on each macula simultaneously, but he projects them $20^{\circ}$ away from each other. This is a very stubborn condition and does not seem to correct itself easily with training. The patient tries to fuse the images and to do so turns the eye back towards the original position of squint and many of the disappointing results of operation are due to this state of the binocular vision.

Where there is a fixed false projection the best procedure is to try to develop true projection and fusion by training and then to operate. Training in an alternating case with a fixed false projection is very tedious unless the treatment is aided by surgical interference.

In this group the accommodation-convergence link is of very secondary value and spectacles cannot be adjusted to be of use in lessening the deviation as in the "refractive error" squints.

\section{Psychological Squints}

This group fulfils Inman's expectation of a patient with squint. The patients are often emmetropic but not invariably so. The age of onset may be early or late in life and the onset of a squint 
after eight years of age, unless there is a definite physical cause, suggests the probability of a psychological basis. The diagnosis and identification of such a condition falls into the province of psychology and it is inevitable that ophthalmologists will identify them with variable accuracy and frequency. Inman finds a history of a psychological cause in the majority of cases, but in my estimate the proportion is nearer 20 per cent. (103 cases out of 500). An analysis of these 103 cases gave the following results :-

Imitative squints, 7 per cent.-These patients gave a history of imitating a beloved parent or friend, or an envied person. In one pair of brothers both squinted, the elder one had a large refractive error, and the young one was emmetropic. The young one squinted a week after the elder one. Squinting competitions are not uncommon at school.

Jealousy squint, 10 per cent.-A frequent cause is the advent of a new baby. The elder child will mope and be unhappy and will suddenly squint. The squint may be constant or only occasional as in the child who squinted when he looked at the baby. The wish seems to be to bring the focus of attention back to the patient.

Fear or shock squints, 26 per cent.-The squint in this group often develops as a direct result of trauma. A child who was frightened at night by a drunken father woke up the following morning with a marked and constant squint.

Difficult children, 28 per cent.-Ten of these were left-handed while the others were of that self-willed type that the psychologist associates with left-handedness. Four of the cases also stammered.

Psychoneurotic parents, 29 per cent.-In these cases the home conditions allowed no peace or liberty for the child to develop a balanced psychological background. One child squinted whenever the father corrected her-the father being an irritable person with very evident psychoneurotic symptoms.

\section{Physical Defect Squints}

Into this group fall those cases in which the squinting eye is irrevocably amblyopic, either congenitally or as the result of injury or disease. The group must include the rare cases of acquired amblyopia which do not react or respond to occlusion or have developed a false macula. It includes also vertical deviations accompanied by torticollis in which there appears to be a defect in the muscles.

It is difficult to assess the relative significance of the factors concerned in the aetiology of squint as there is so much overlapping and two factors may have an equal value. Nevertheless, grouping based on the classification above has been found useful and of practical value especially in prognosis. 